\title{
Selection of Energy and Queue Aware Forwarding Neighbor in Wireless Mesh Network
}

\author{
V.Lakshmi Praba \\ Asst. Professor \\ Govt.Arts College for Women \\ Sivaganga, India
}

\author{
A.Mercy Rani \\ Research Scholar \\ Manonmaniam Sundaranar University \\ Tirunelveli, India
}

\begin{abstract}
Wireless Mesh Network has more attractive features such as high-speed last-mile wireless internet access facility, instant deployability and low-cost backhaul services for large area coverage, etc. These features increase the application areas of Wireless Mesh Network (WMN) which in turn, persuade the researchers to pay more attention on this field. Routing plays a vital role in WMN for providing the Quality of Service (QoS) to the users in the network. In this paper, an EQ-AODV protocol is proposed to select a forwarding neighbor based on the factors such as node's maximum remaining energy and minimum queue length. The above mentioned factors can be incorporated in the multi-channel and multi-interface approach of WMN to increase the capacity and throughput of the network. The EQ-AODV protocol constructs a stable and unbiased traffic route by considering the above mentioned factors. The analysis was carried out by comparing the proposed protocol EQ-AODV with the existing AODV and with the two variations of AODV such as AODV with maximum energy (MaxEnergy) and AODV with minimum queue length (MinQueue). The performance was evaluated in terms of packet delivery ratio, dropped packets, routing overhead, average end-end delay and energy consumed per packet.
\end{abstract}

\section{General Terms}

Computer Science - Network - Wireless Mesh Network

\section{Keywords}

Minimum Queue, Unbiased Traffic, Energy Consumption, Maximum Energy, Forwarding Neighbor and Throughput

\section{INTRODUCTION}

Wireless Mesh Network is a promising technology in the wireless world for its abundant applications such as broadband home networking, enterprise networking, community and neighborhood networks etc. WMN has the advanced features like low deployment cost, easy network maintenance, robustness, resilent, wide area network coverage, self-healing, self-configuring, self-organizing and also provides quality broadband wireless access to the Internet[1][2]. Due to these features, WMN is primarily appropriate for Home Automation, Healthcare, Industries, Disaster recovery and Historical Monuments [3].

Wireless Mesh Network is a communication network made up of radio nodes arranged in mesh topology. In mesh topology all the nodes are connected to more than one node in the network. WMN often consists of mesh clients, mesh routers and gateways. The mesh clients can be laptops, cell phones, stationary nodes or other wireless communication devices. The mesh routers are static and it forwards traffic to and from the gateways. The gateways may or may not be connected to the Internet. The topology in the mesh network changes frequently because the mobile nodes are dynamically connected with one another [1].

The existing on-demand routing protocols AODV (Adhoc OnDemand Distance Vector) [4] and DSR (Dynamic Source Routing) [5] are designed to find the route based on the single factor hop count without considering the other network factors like node's energy, queue length and link quality etc. The recent applications of WMN such as Disaster recovery, High speed last mile Internet access, Video Conferencing and lowcost backhaul services for large area coverage requires Quality of Service (QoS) for supporting effective communication in the network. This can be achieved by selecting the forwarding neighbor with more than one factor. The factors such as node's energy and queue length are important for selecting the forwarding neighbor since it increases the route's lifetime and decreases the packet drops to a greater extent. The node in the network depletes its energy during the transmission for sending as well as for receiving the packets. The queue length indicates the load of the node during the packet transmission.

The minimal energy route is considered for the transmission to reduce the total energy consumption of the network [6]. The problem arises in this method is, if more than one traffic choose the same minimum energy node for their transmission, it leads to node congestion and the energy of a node decreases rapidly. This causes the node to get disconnected from the route very quickly so that it increases the route discovery latency. The increase of route discovery latency degrades the performance of the network considerably [7]. In addition to that the nodes with maximum energy and minimum queue length are not participated in the route selection process. Hence, the network traffic becomes unbalanced because of the existence of idle nodes with maximum energy and busy nodes with minimum energy. The busy nodes with higher queue length leads to congestion, packet drops, long delays and the unbalanced traffic load. The performance of the network can be improved if the selected route has a maximum energy [8] and minimum queue length [9]. This paper modifies an existing routing protocol AODV to propose an EQ-AODV protocol by considering the maximum energy as well as the minimum queue length nodes for the route construction.

Traditional routing protocols for wireless networks assume that each node is equipped with one interface. An interface is a network interface card equipped with a half-duplex radio transceiver. The routers in the mesh network are provided with multiple interfaces increase the network throughput considerably [10]. A channel is a wireless spectrum with a specified bandwidth. In infrastructure-based wireless 
networks, multiple channels are incorporated by assigning different channels to adjacent mesh routers for minimizing interference. The usage of multiple channels and multiple interfaces increase the network throughput and provide concurrent transmissions. The EQ-AODV protocol constructs the route by considering the above mentioned factors (energy and queue) by means of incorporating the multi-channel and multi-interface approach [11] in the considered wireless mesh network architecture.

The paper is organized as follows: Section 2 deals with related work, section 3 describes an energy and queue aware route selection process and section 4 depicts simulation process and results. The conclusion and future scope is discussed in section 6 .

\section{RELATED WORK}

Qutaiba et al[8] proposed a new protocol MEP-AODV which considers path energy as an additional metric for route selection. Dhiraj et al[12] developed a new routing algorithm ECNC_AODV by considering the current energy status of each node and the cached node. The current node's energy is compared with energy threshold. If it is above the threshold then the node will forward the packet otherwise it will drop the packet. Hongkun et al[13] studied the selection of a path with the minimum cost in terms of expected end-to-end delay (EED) in a multi-radio wireless mesh network. The new EED metric takes the transmission delay as well as the queuing delay of the buffer. The EED metric can be decreased by the concept of load balancing and also the EED based routing protocols were designed for both single-channels as well as multi-channel wireless mesh networks.

Yumei et al[13] proposed a MMRE-AOMDV routing protocol which exploited maximal minimal nodal residual energy concept. The proposed protocol balanced the nodal energy consumption. This protocol found the minimal nodal residual energy of each route in the route discovery process, then sorts the multi route by descending nodal residual energy and selected the route with maximal residual energy to forward the data packets. Yajun et al[14] presented an optimization architecture for joint multi-path QoS routing and the underlying wireless link scheduling in wireless mesh networks. The proposed work used the contention matrix to represent the wireless link interference and formulate a utility maximization problem for the joint multi-path routing and MAC scheduling and resolved it using the primal_dual method. In addition to that, the proposed work has an algorithm to solve the routing sub problem and the scheduling sub problem through the dual decomposition. The simulations demonstrated the efficiency and correctness of the proposed algorithm.

Marija et al[15] analyzed the problem of selecting optimal paths in a MCMI (Multi-Channel Multi-Interface) WMN. The three new metrics such as ETX, WCETT and MIC were compared against known metrics in terms of throughput of user data and average end-to-end delay of the network. Yanbing et al[16] analyzed the average queuing delay, average queuing length, and optimize the average queuing delay by increasing the number of network interfaces as well as the transmitting rate of sending interfaces. Capdehourat et al[17] proposed an algorithm for dynamic multipath forwarding in a WMN. The algorithm enabled load balancing and carries out the network to operate at the minimum average congestion. The proposed framework also solved the gateway selection problem by learning the average queue length function of each link and applying an optimization method to get the minimum average queue length in the network

Ma Liang et al[18] proposed a routing metric known as Weighted Cumulative Expected Transmission Time with Load-Balancing (WCETT-LB) for wireless mesh networks. WCETTT-LB enhanced the basic Weighted Cumulative Expected Transmission Time (WCETT) by incorporating load-balancing into the routing metric. WCETT-LB provided a congestion-aware routing and traffic splitting mechanism to achieve global load-balancing in the network. Mohammed et al[19] modified the AODV routing protocol to combine the route discovery process and the load balancing technique. The modified protocol selected a route to the destination based on the current load of the intermediate nodes and selected a gateway from the available mesh network gateways based on its current load. The simulations showed that this load balancing technique improved the performance of the wireless mesh networks.

The researchers were considered only the energy or the queue length for the route selection. In this paper, the two factors node's energy and queue length are considered for selecting an efficient route with unbiased traffic to further improve the performance of the network.

\section{EQ-AODV - Energy Queue Aware Route Selection Process}

The proposed EQ-AODV protocol selects the forwarding neighbor with maximum energy and minimum queue length. The main idea behind this proposed protocol design is, even if it considers maximum energy node for transmitting the packets, it consumes less energy for a single packet transmission.

The each node in the network is equipped with multiple interfaces and each interface can operate on any one of the multiple non-overlapping channels. When a route is required, the RREQ packet is broadcasted simultaneously on all the interfaces. The neighbors who share at least one common channel with the sender node can receive the packet. The routing table of this modified protocol contains an interface number [11] to indicate through which the next hop node will be reached for a selected/particular route.

RREQ and RREP are the control packets used by AODV protocol to discover the route in the network. In EQ-AODV protocol the RREQ packet is modified with three additional fields RREQ_ENERGY, RREQ_QLEN and SEL_NBR and the RREP packet with two additional fields RREP_ENERGY and RREP_QLEN which is shown in Fig.1. RREQ_ENERGY field in RREQ packet has been assigned the ENERGY_THRESHOLD and RREQ_QLEN has been assigned the node's current queue length. ENERGY_THRESHOLD is assigned a minimum energy value for a node to transmit the packets successfully. SEL NBR field will have the neighbor's node-id which acquires the maximum remaining energy as well as the minimum queue length among all the neighbors. RREP_ENERGY field in RREP packet holds their remaining energy and REP_QLEN field holds their current queue length when the RREP packet is sent.

\begin{tabular}{|l|l|l|l} 
Existing fields & RREQ_ENERGY & RREQ_QLEN & SEL_NBR
\end{tabular} 


\section{\begin{tabular}{|l|l|l|} 
Existing fields & RREP_ENERGY & RREP_QLEN \\
\hline
\end{tabular}}

Fig1.2 Additional Fields in RREP Packet

The EQ-AODV protocol has the following modifications.

- Neighbors List Maintenance.

- Constructs the Stable and Unbiased Traffic Route - by selecting the forwarding neighbor with Maximum Energy and Minimum Queue.

\subsection{Neighbor List Maintenance}

To maintain the neighbor's details of each node, the node sends a link-layer feedback or periodic HELLO packets to nodes which are considered as its direct neighbors. The neighbors keep the information such as cur_node_id, nbr-id, its remaining energy and current queue length. In case of a link break during the next hop of an active route, a RERR packet is sent to its active neighbors.

\subsection{Selection of Stable and Unbiased Traffic Route}

This can be achieved by selecting the forwarding neighbor with Maximum Energy and Minimum Queue. Before broadcasting the RREQ, the source/forwarding node calls the procedure OPT_NBR(). The procedure initially finds the neighbors of the current node, checks the remaining energy of all its neighbors and selects only the neighbors which have the energy value above the ENERGY_THRESHOLD. After that it finds the neighbor which has maximum energy as well as the minimum queue length. The selected neighbor ID is stored in the SEL_NBR field of RREQ packet. The following formula shows the neighbor selection process of EQ-AODV.

$\operatorname{SEL} \_N B R=\left\{\operatorname{NBR} . i d \mid\left(\operatorname{Max}\left(\mathrm{E}_{\mathrm{R}}\left(\mathrm{y}_{\mathrm{j}}\right)\right)\right) \& \& \quad\left(\operatorname{Min}\left(\mathrm{Q}_{\mathrm{L}}\left(\mathrm{y}_{\mathrm{j}}\right)\right)\right)\right.$ $\left.\left(\exists \mathrm{y}_{\mathrm{j}}\left(\forall \mathrm{x}_{\mathrm{i}} \in \mathrm{NBR}\left(\mathrm{E}_{\mathrm{R}}>\mathrm{E}_{\mathrm{TH}}\right)\right)\right)\right\}$

\section{$1 \leq \mathrm{i} \leq \mathrm{k}, 1 \leq \mathrm{j} \leq \mathrm{l}$}

$\left(\mathrm{E}_{\mathrm{R}}-\right.$ Remaining Energy, $\mathrm{E}_{\mathrm{TH}}$-Energy Threshold, $\mathrm{Q}_{\mathrm{L}}-$ Queue Length, $\mathrm{k}$ is the number of neighboring nodes; 1 is the number of nodes that are above $\mathrm{E}_{\mathrm{TH}}$ )

The source node broadcasts a RREQ to its neighbors. The neighbors receive the RREQ and checks whether it is a destination, if it is so it sends RREP back to the sending node. If else the remaining energy of a node has to be checked. If the energy is below the RREQ_ENERGY, it discards the packet. If it is not a destination and its energy is beyond the RREQ_ENERGY, the SEL_NBR is checked to find the match with the current node-id. If it matches, it forwards the RREQ to its neighbors. This process will be repeated until the destination is reached.

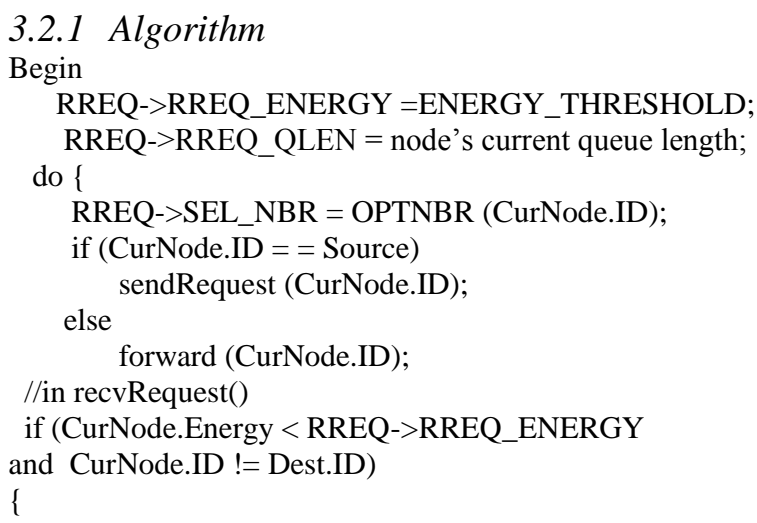

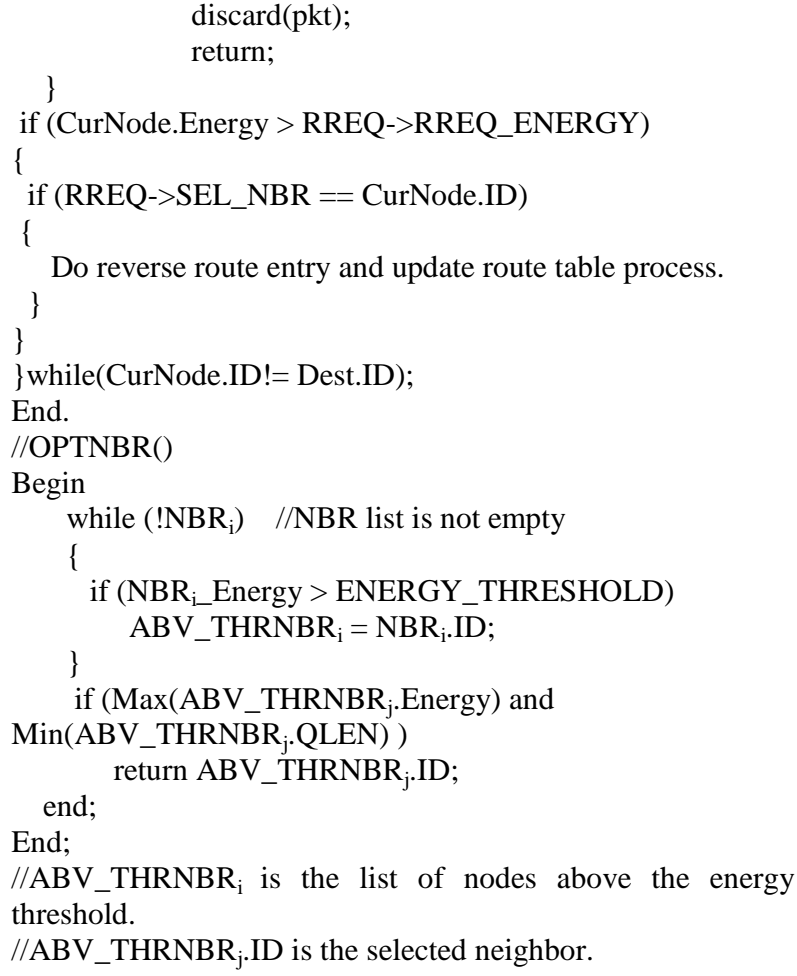

When the destination is reached the remaining energy as well as the queue length of the current node is assigned to RREP_ENERGY and RREP_QLEN fields of RREP packet. The further process is similar to AODV. The route is made available by unicasting a RREP back to the origination of the RREQ. Each node receiving the request caches a route back to the originator of the request, so that the RREP can be unicast from the destination along a path to that originator, or likewise from any intermediate node that is able to satisfy the request. In this way, the EQ-AODV protocol selects a stable and unbiased traffic route for transmitting the packets from the source to the destination.

\section{SIMULATION PROCESS \& RESULTS}

The simulations are carried out using NS-2[20]. For the performance appraisal of EQ-AODV protocol in WMN, a network model with 1 Gateway, 48 Mesh Routers and 10 Mesh Clients has been created. The network is structured as a grid of size $7 \times 7$ and each node is separated from the other by a distance of $100 \mathrm{~m}$.

The initial energy is 100 joules with transmission and receiving power of $35.28 \mathrm{e}-3 \mathrm{~W}$ and $31.32 \mathrm{e}-3 \mathrm{~W}$ respectively. The simulation parameters are shown in Table 1 . The mesh clients and mesh routers are placed in an area of $800 \times 800$ meters. The mesh routers are sited statically so that it helps the mesh clients in establishing reliable connections to the mesh routers and to the other mesh clients. The Simulation Layout is shown in Fig.2. The CBR connections are created to establish the connection. The simulation is performed under the varying number of traffic flows. 
Table 1: Simulation Parameters

\begin{tabular}{|l|l|}
\hline \multicolumn{1}{|c|}{ Parameter } & Value \\
\hline Simulation & NS-2 \\
\hline Simulation area & $800 \times 800 \mathrm{~m}$ \\
\hline Mac Protocol & IEEE 802.11 \\
\hline Simulation time & $200 \mathrm{~s}$ \\
\hline Transmission range & $200 \mathrm{~m}$ \\
\hline Data Rate & $9 \mathrm{Mb}$ \\
\hline Packet Size & 512 bytes \\
\hline Transmission rate & $1 \mathrm{Mb}$ \\
\hline Traffic Type & CBR(UDP) \\
\hline No. of Mesh Clients & 10 \\
\hline No. of Mesh Routers & 48 \\
\hline No. of Gateway & 1 \\
\hline Routing Protocol & AODV \\
\hline Traffic Model & CBR \\
\hline Interfaces & 2 \\
\hline
\end{tabular}

\subsection{Performance Metrics}

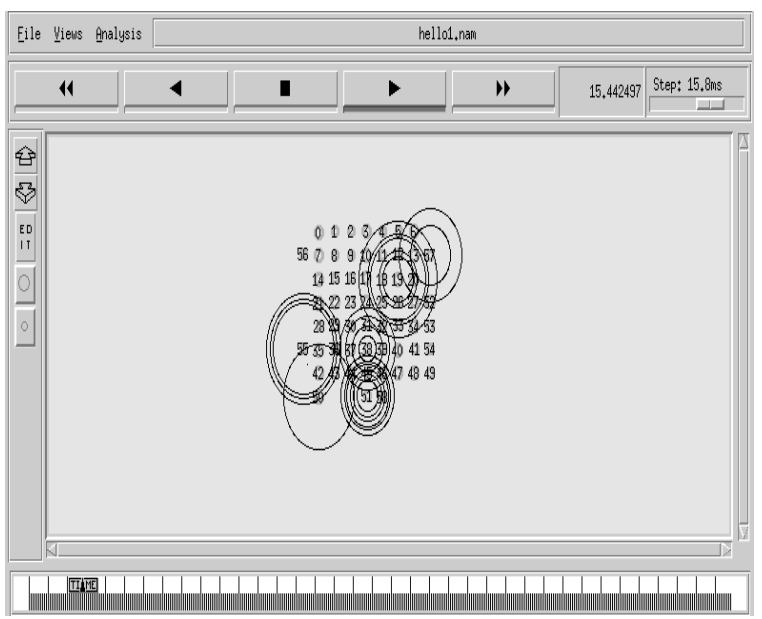

Fig 2. Simulation Layout

This paper considers the Packet Delivery Ratio(PDR), dropped packets, routing overhead, average end-end delay and energy consumed per packet as performance metrics which were analyzed by varying the number of traffic flows.

\subsubsection{Packet Delivery Ratio (\%):}

Received packets/Sent packets * 100

\subsubsection{Dropped Packets}

Sent packets - Received packets

\subsubsection{Routing overhead}

Routing packets/Received packets

\subsubsection{Average End-End Delay}

(End-time - Start time) / Total No. of Packets Generated

\subsubsection{Energy Consumed/Packet}

Total Energy Used/Total No. of Packets received

\subsection{Simulation Results}

The analysis was illustrated by comparing the proposed protocol EQ-AODV with the two variations of AODV and the existing AODV. The two variations are MaxEnergy protocol which constructs the stronger route with maximum energy and the MinQueue protocol which constructs the low-traffic and congestion-free route by considering the minimum queue length.

These modified protocols were compared with AODV in terms of the above considered performance metrics. Any protocol which aims a better QoS should achieve maximum PDR and minimum number of dropped packets. Similarly, average end to end delay and routing overhead has been minimized. Also, low energy consumption plays a vital role particularly with mobile devices. All these QoS parameters were analyzed and obtained results are depicted in Figure.3.1a to 3.1e. From the obtained results, it is very clear that EQAODV satisfies the QoS parameters and brings out better performance than MaxEnergy, MinQueue and AODV.

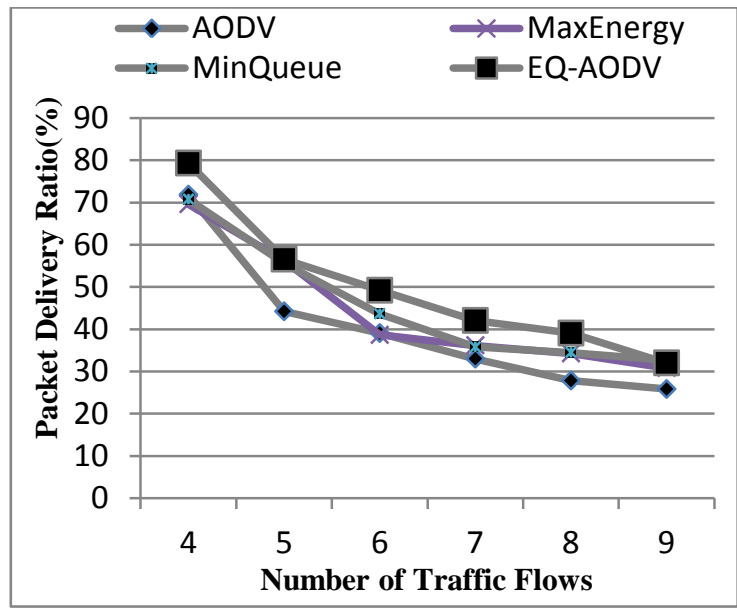

Fig 3.1a Packet Delivery Ratio Vs Traffic Flows

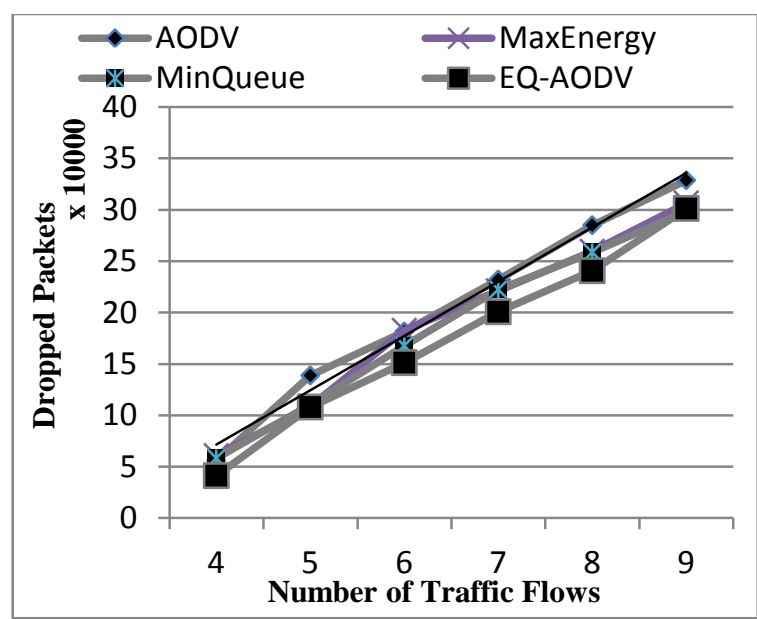

Fig 3.1b Dropped Packets Vs Traffic Flows 


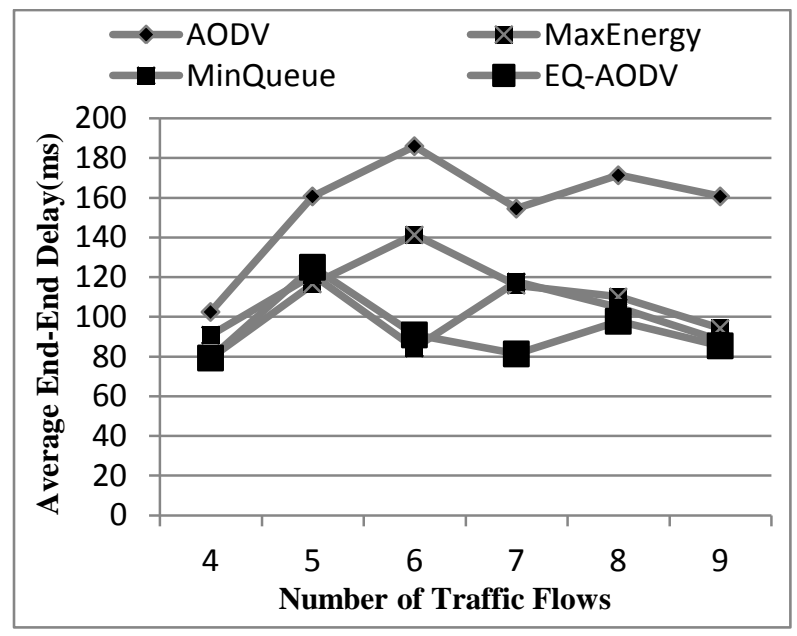

Fig 3.1c Average End-End Delay Vs Traffic Flows

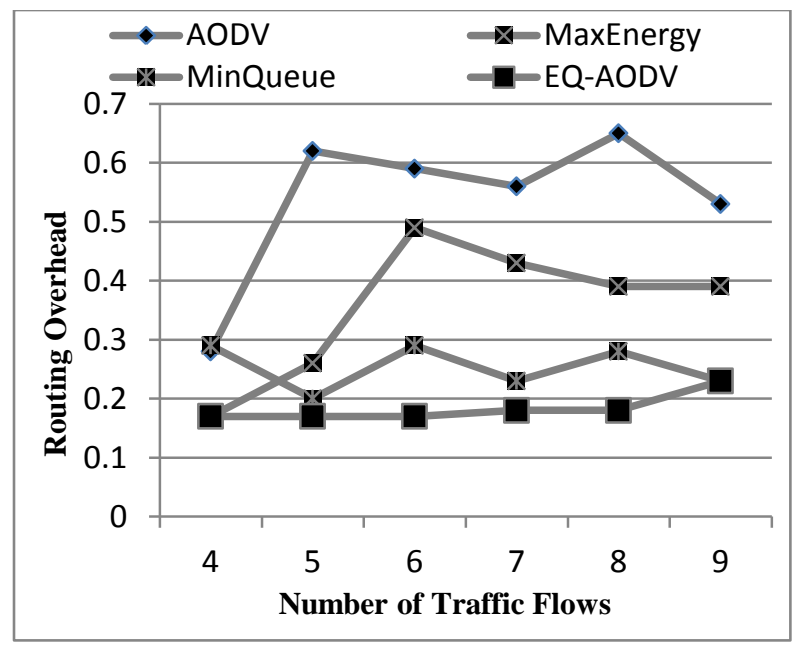

Fig 3.1d Routing Overhead Vs Traffic Flows

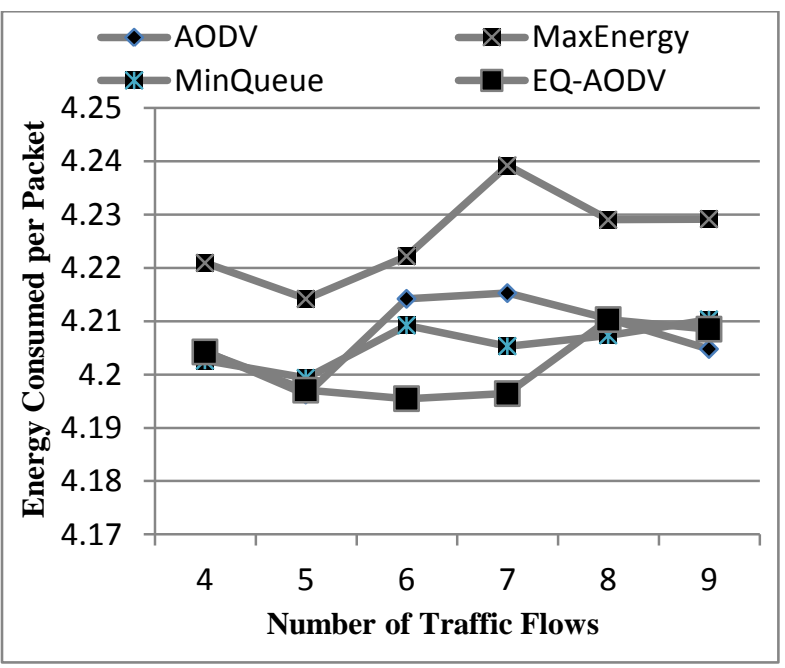

Fig 3.1e Energy Consumed per Packet Vs Traffic Flows

\section{CONCLUSION}

Wireless Mesh Network is a promising area for the wide range of applications due to their attractive features. Efficient route construction is an important issue to be considered in WMN. In this paper, an EQ-AODV protocol is proposed to construct an efficient route by selecting the forwarding neighbor with maximum energy and minimum queue length. The proposed protocol also constructs the route by satisfying the QoS parameters such as maximum PDR, least amount of packet drops, less delay, reduced routing overhead and low energy consumption. The better QoS parameters provided the EQ-AODV to reduce the congestion as well as route discovery latency and to increase the route lifetime. Furthermore, the protocol provides better throughput and increased network capacity with an additional support of multi-channel multi-interface approach. A fascinating point which could be more deeply studied in the future is the optimization process.

\section{REFERENCES}

[1]. Akyildiz, I.F and Xudong Wang, "A survey on wireless mesh networks, Communications Magazine", IEEE, vol.43, , Sept. 2005, pp.S23 - S30.

[2]. http://en.wikipedia.org/wiki/Wireless_mesh_network

[3]. "What's so good about mesh networks?" Daintree Networks, www.daintree.net, Jan. 2007.

[4]. Perkins C.E. and Royer E.M, "Ad hoc on demand distance vector (AODV) routing" (Internet-draft), in: Mobile Ad-hoc Network (MANET) Working Group, IETF (1998).

[5]. David B. Jhonson, David A.Maltz and Josh Broch, "DSR: The Dynamic Source Routing protocol for MultiHop Wireless Adhoc Networks", www.monarch.cs.rice.edu/monarch-papers/dsrchapter00.pdf.

[6]. Yumei Liu, Lili Guo, Huizhu Ma and Tao Jiang, "Energy efficient on demand multipath routing protocol for multihop ad hoc networks", in the Proceedings of ISSSTA08, IEEE 10th International symposium on Spread spectrum and applications, Bologna, Italy, August 25-27 2008, pp-592-597.

[7]. Jasem and Hayder Natiq, "On the delay and link utilization with the new-additive increase multiplicative decrease congestion and control algorithm", Scientific Research and Essays, vol. 5(23), December 2010, pp. 3719-3729.

[8]. Qutaiba A. Razouqi and Ali H. Afsari, "MEP-AODV: AODV-Based Maximum Energy Path Routing in Ad Hoc Networks" in proceedings of ISCCSP, Marrakech, Morocco, March 2007.

[9]. Yunxia Feng, Xinfa Shen, Zhigang Gao and Guojun Dai, "Queuing Based Traffic Model for Wireless Mesh Networks", in $15^{\text {th }}$ International Conference, Shenzhen, China, Dec. 2009, pp. $648-654$.

[10]. Guokai Zeng, Bo Wang, Yong Ding,, Li Xiao and Matt W. Mutka, "Efficient Multicast Algorithms for Multichannel Wireless Mesh Networks" , IEEE Transactions On Parallel And Distributed Systems, Vol. 21, No. 1, January 2010, pp-86-99. 
[11]. Ramon Aguero Calvo and Jesus Perez Campo, "Adding Multiple Interface Support in NS-2", http://personales.unican.es/aguerocr, Jan 2007.

[12]. Dhiraj Nitnaware and Ajay Verma, "Energy Constraint Node Cache Based Routing Protocol for Adhoc Network", International Journal of Wireless \& Mobile Networks (IJWMN), Vol. 2, No.1, February 2010, pp 77 86.

[13]. Hongkun Li, Yu Cheng, Chi Zhou and Weihua Zhuang, "Minimizing End-to-End Delay: A Novel Routing Metric for Multi-Radio Wireless Mesh Networks", IEEE, INFOCOM 2009, pp:46 - 54.

[14]. Yajun Li, Liang Zhou, Yuhang Yang and Han-Chieh Chao, "Optimization architecture for joint multi-path routing and scheduling in Wireless Mesh Networks", Elsevier, Mathematical and Computer Modelling,53(3), 2011, pp. 458-470.

[15]. Marija Malnar, Natasa Neskovic and Aleksandar Neskovic, "Novel power-based routing metrics for multichannel multi-interface wireless mesh networks", Springer, Wireless Networks, May 2013, pp.1-11.
[16]. Yanbing Liu, Tao Wu and Xingping Xian, "Queue control based delay analysis and optimization for wireless mesh networks', Education Technology and Computer (ICETC), 2nd International Conference on June 2010, Vol 1, pp. V1-104 - V1-108.

[17]. Capdehourat, Germán, Federico Larroca, and Pablo Belzarena. "Minimum queue length load- balancing in planned Wireless Mesh Networks." In Wireless Communication Systems (ISWCS), International Symposium, IEEE, 2012, pp. 781-785.

[18]. Ma, Liang, and Mieso K. Denko. "Enhanced Routing Metric for Load-Balancing in Wireless Mesh Networks." Journal of Interconnection Networks, 8, no. 04, 2007, pp. 407-426.

[19]. Mohammed I. Gumel, Nasir Faruk and A.A. Ayeni, "Routing with load balancing in wireless mesh networks, International Journal of Current Research, Vol. 3, July, 2011, pp.087-092.

[20]. NS-2 Network Simulator http://www.isi.edu/nsnam/ns. 